\section{Targeting genes for self-excision in the germ line}

\author{
Michaeline Bunting, ${ }^{1}$ Kenneth E. Bernstein, ${ }^{2}$ \\ Joy M. Greer, ${ }^{3}$ Mario R. Capecchi, ${ }^{3}$ \\ and Kirk R. Thomas ${ }^{3,4}$

\begin{abstract}
${ }^{1}$ Hematology Division, Department of Internal Medicine, University of Utah, Salt Lake City, Utah 84112 USA; ${ }^{2}$ Department of Pathology, Emory University, Atlanta, Georgia 30322 USA; ${ }^{3}$ Howard Hughes Medical Institute, Department of Human Genetics, University of Utah School of Medicine, Salt Lake City, Utah 84112 USA
\end{abstract}

A procedure is described that directs the self-induced deletion of DNA sequences as they pass through the male germ line of mice. The testes-specific promoter from the angiotensin-converting enzyme gene was used to drive expression of the Cre-recombinase gene. Cre was linked to the selectable marker $\mathrm{Neo}^{\mathrm{r}}$, and the two genes flanked with $\operatorname{lox} P$ elements. This cassette was targeted to the Hoxa3 gene in mouse ES cells that were in turn used to generate chimeric mice. In these chimeras, somatic cells derived from the ES cells retained the cassette, but self-excision occurred in all ES-cell-derived sperm.

Received April 16, 1999; revised version accepted May 3, 1999.

The paradigm for targeted germ-line modification of a mammalian genome was established 12 years ago (Thomas and Capecchi 1987). An alteration introduced in vitro into a cloned gene is transferred by homologous recombination to its chromosomal target in a pluripotent embryo-derived stem (ES) cell. Cells containing the modification are placed in an embryonic environment, allowed to grow, differentiate, and contribute to the germ line of the host organism. Limitations imposed by the transformation and recombination efficiencies of mammalian cells require that the alteration of interest be linked physically to a selectable genetic marker, typically a gene encoding drug resistance under transcriptional control of a constitutive promoter/enhancer element. This operational requirement can have unpredictable consequences in vivo, such as misregulation of adjacent genes (Olson et al. 1996) or the attenuation of expression of the gene of interest (Colledge et al. 1995; Meyers et al. 1998). Thus, the elimination of the marker may be desirable and, for technical reasons, is generally performed through use of site-specific recombinase systems such as Cre/loxP (Sternberg and Hamilton 1981) or FLP/FRT (Broach and Hicks 1980). To facilitate this pro-

[Key Words: Targeted gene modification; conditional mutagenesis; gene therapy; Cre/loxP; angiotensin-converting enzyme]

${ }^{4}$ Corresponding author.

E-MAIL thomas@howard.genetics.utah.edu; FAX (801) 581-3770. cess, we have devised a self-excision procedure that takes advantage of a testes-specific promoter to drive expression of the Cre recombinase. The marker gene, linked to Cre, survives selection in cultured cells, but is removed along with Cre as both are passed through the male germ line.

The strategy behind this protocol is illustrated in Figure 1: The intragenic promoter of the murine angiotensin-converting enzyme, tACE (shown to initiate transcription only during spermatogenesis; Langford et al. 1991), directs expression of Cre; $t A C E-C r e$ is linked to the selectable marker gene $\mathrm{NeO}^{\mathrm{r}}$, and the two genes tACE-Cre $/ \mathrm{NeO}^{\mathrm{r}}$, are flanked with lox $\mathrm{P}$ sites. This cassette, referred to as ACN, is targeted by homologous recombination to a specific locus in a murine ES cell. Cells containing the appropriate chromosomal recombinant are inserted into a blastocyst-stage mouse embryo that develops into a chimeric animal, containing cells from both the host blastocyst and the cassette-containing ES cells. If the chimerism extends to the germ line of an adult male, some fraction of the sperm will be ES-cell derived. During spermatogenesis the tACE promoter induces expression of the Cre-recombinase gene, the ACN cassette is excised, and a single lox $P$ element remains at the chromosomal locus. Progeny from these sperm should represent two classes of paternal transmission: (1) those containing a wild-type paternal chromosome, originating either from the nontargeted chromosome in the heterozygous ES cells or from non-ES (i.e., host)-derived cells; and (2) those containing a loxP insertion in the paternal chromosome.

\section{Results and Discussion}

The experimental design used to test this protocol is illustrated in Figure 2. Two features of the ACN cassette are noteworthy: $N e O^{\mathrm{r}}$ is located $3^{\prime}$ of the $t A C E-C r e$ gene, such that transcription of $\mathrm{NeO}^{\mathrm{r}}$ should not result in transcriptional readthrough of Cre; and the Cre gene contains an intron to prevent in-frame translation and subsequent self-excision in bacteria. We inserted the ACN cassette into a genomic clone of the mouse Hoxa3 gene (McGinnis et al. 1984) and transfected the targeting vector into mouse ES cells. We clonally isolated 144 cell lines that survived positive/negative selection and demonstrated by Southern transfer analysis that 20 contained the ACN cassette integrated into one of the endogenous Hoxa3 loci.

Three of the recombinant ES cell lines were used to generate 13 male chimeric mice that, in turn, sired 138 ES cell-derived progeny (determined by coat color). All progeny were genotyped by a PCR-based assay that could distinguish between the three potential Hoxa3 alleles: wild type, ACN, and loxP (Fig. 3a). Figure 3b shows such an assay, comparing DNA isolated from the parental ES cell line, one recombinant ES cell line, tail biopsies from a chimeric male, and six of his agouti progeny. The recombinant ES cells and the chimera-derived tails are het- 
A

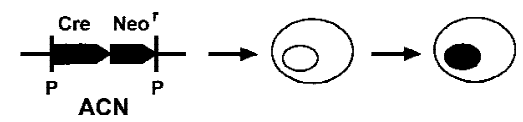

B

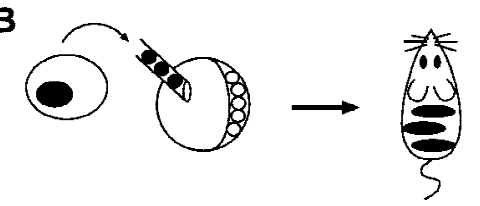

C

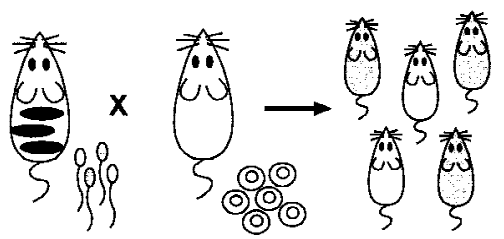

Figure 1. Testes-specific self-excision. $(A)$ A selectable marker gene, $N e o^{\mathrm{r}}$, with a constitutive promoter, is transferred by homologous recombination to a specific locus in a mouse ES cell. The $N e o^{r}$ gene is linked to a Cre gene that is under transcriptional control of the tACE promoter, and the two genes are flanked with $\operatorname{lox} P$ sites $(\mathrm{P})$; the entire cassette, $\mathrm{ACN}$, is introduced by gene targeting to a specific locus in a mouse ES cell. (B) ES cells, heterozygous for an allele containing the integrated cassette, are injected into wild-type mouse blastocysts and the blastocysts allowed to develop; the resulting animals are chimeric for wild-type (host-derived) cells (white) and ES-derived cells (black). (C) Male chimeric animals will transmit through their sperm one of two alleles of the locus of interest: wild-type (white) or mutant (gray); if self-excision has occurred the mutant allele will be marked only by a $\operatorname{lox} P$ site, the final product of the testes-specific self-excision reaction.

erozygous for the wild-type and ACN-containing alleles, whereas the $\mathrm{F}_{1}$ progeny are either wild type or heterozygous for the loxP allele. A summary of the genotypes of all 138 progeny, shown in Table 1, demonstrates that tACE-Cre mediated germ line excision of the ACN cassette in all cases.

Although self-excision was complete at the level of spermatogenesis, it was also restricted to the testes. Tissues from chimeric males that transmitted the $\operatorname{lox} P$ allele were genotyped and, with the exception of the testes, were heterozygous for wild-type and ACN alleles (Fig. 3c). Testes, which were mosaic for the two mutant Hoxa3 alleles, include multiple cell types, only two of which, the elongating spermatids and the spermatozoa, should contain the loxP allele (Langford et al. 1991).

A similar protocol has been used to generate mice carrying a loxP insertion in the Hoxd3 gene (data not shown), and it should be applicable to a vast number of loci. The tACE promoter is inactive in somatic cells when integrated at independent ectopic sites (Langford et al. 1991). It also appears refractory to activation when integrated at random loci in ES cells, even when linked to a transcriptionally active $N e o^{r}$ gene. Were the tACE promoter expressed frequently following integration in ES cells, the capacity of DNA carrying the self-excision cassette to generate stable transformants would be greatly reduced, but this is not the case (data not shown). It remains possible, however, that if the cassette were targeted to a transcriptionally active locus, the Cre protein could be translated from readthrough mRNA transcribed into the cassette. Under such conditions, it would be necessary to custom-design a cassette containing transcription insulators (Pikaart et al. 1998) or to place ACN in a transcriptional orientation opposite that of the target locus.

A

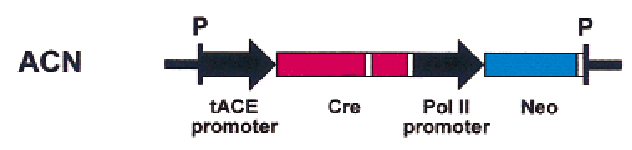

B

C
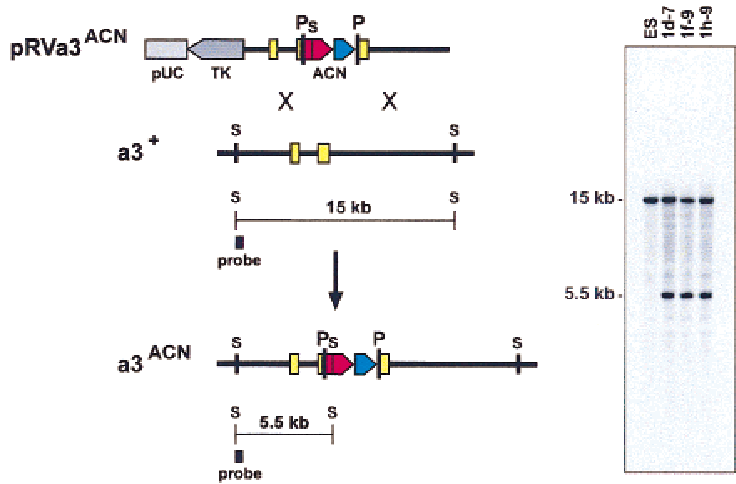

Figure 2. Targeting of a self-excision cassette to Hoxa3. (A) Self-excision cassette, ACN: The testes-specific elements from the mouse ACE gene (black arrow) are placed 5' of the modified Cre structural gene (Gu et al. 1993) (red), followed, 3', with the minimal polyadenylation signal from $H S V-T K$ (Thomas and Capecchi 1987) (white box); an intron, derived from the SV40 $t$ antigen gene (white box), is inserted into the Cre gene; the $\mathrm{NeO}^{\mathrm{r}}$ gene (blue) is controlled by a promoter from the mouse RNA polymerase II gene (black arrow) and followed also by the HSVTK poly(A) site (white box). The $5^{\prime}$ and $3^{\prime}$ ends of this cassette contain loxP sites (P). (B) Gene targeting at Hoxa3: (Top line) The targeting vector $\mathrm{pRVa} 3^{\mathrm{ACN}}$; the vector contains $11 \mathrm{~kb}$ of mouse genomic DNA into which the self-excision cassette ACN has been inserted in the homeodomain of Hoxa3 (McGinnis et al. 1984); the genomic sequences are linked to the HSV$T K$ gene (dark gray) and all are contained on a pUC-based plasmid backbone (light gray); the ACN cassette contains at its 5' end an SstI site (S), used as a marker for homologous integration of the cassette at the Hoxa3 gene; (second line) the wild-type Hoxa3 locus; (bottom line) the predicted structure of the recombinant $\mathrm{Hoxa}^{A C N}$ allele. The $5^{\prime}$ flanking probe used to detect recombination is indicated, and the diagnostic SstI-generated DNA fragments delineated beneath each locus. Yellow boxes designate Hoxa3 exons; other SstI sites in the vector are not indicated. $(C)$ Southern transfer analysis: DNA from the parental cell line (ES) and the homologous recombinant ES lines used to generate mice was restricted with SstI; radiolabeled DNA probe is depicted in $B$. 
A

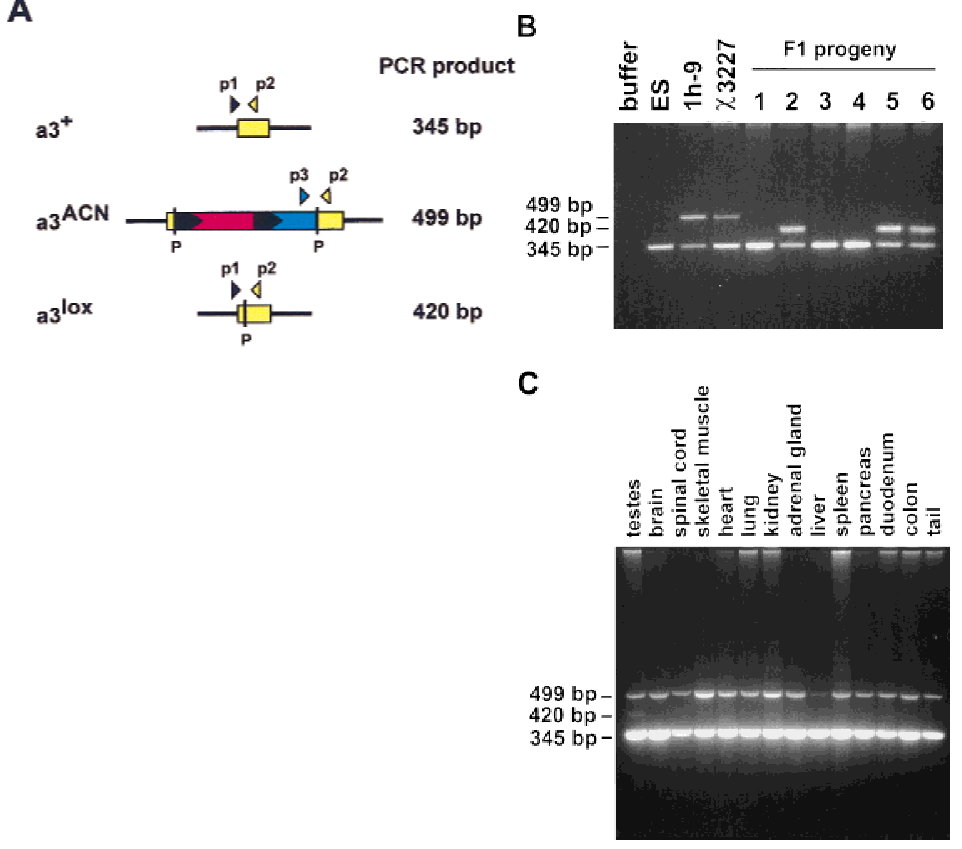

Figure 3. Genetic transmission of Hoxa3 alleles. (A) The PCR-based genotyping of the three Hoxa3 alleles: Primer $1(\mathrm{p} 1)$ is from the Hoxa3 intron; primer 2 (p2) is from coding exon 2-derived sequences (antisense); primer $3(\mathrm{p} 3)$ is from the $N e \mathrm{O}^{\mathrm{r}}$ gene. Predicted sizes are indicated; color coding is as in Fig. 2. $(B)$ Genotyping of DNA: DNA is from wild-type ES cells (ES), recombinant ES cell line, 1h-9, tail biopsies from a chimeric male, $\chi 3227$, generated from $1 \mathrm{~h}-9$, and tail tissue from $\mathrm{F}_{1}$ progeny of the chimera; amplified DNA was electrophoresed through agarose and stained with ethidium bromide. Sizes correspond to those listed in $a .(C)$ Absence of excision in somatic tissue. A single chimeric male derived from cell line 1h-9 was sacrificed at 8 weeks of age. DNA extracted from each of the indicated tissues was analyzed by PCR as in B. Analysis of a second chimera showed an identical result.

Potential uses for a self-excision cassette such as the one described here are numerous. In the generation of knockout animals, this technology should see immediate application. The possibility that a marker gene may affect phenotype unpredictably has already prompted removal of such sequences prior to phenotypic analysis (Hasty et al. 1991; Meyers et al. 1998). Although alternative recombinase-based excision methods do exist, they are often accompanied with operational inconveniences. For example, removal of sequences during the growth of ES cells (Gu et al. 1993) requires additional selection and/or screening. Not only is there a time and labor consideration involved in such manipulation, but the pluripotency of ES cells can be adversely affected by prolonged growth in culture. Sequence deletion in the animal relies either on the expression of the recombinase in the fertilized eggs of animals carrying a lox $P$-flanked gene (Araki et al. 1995), the mating of such animals with a Cre-expressing mouse (Schwenk et al. 1995), or the use of ES cells containing a Cre-expressing transgene (O'Gorman et al. 1997). All methods require additional breeding and/or technical expertise, and thus prolong by several months the time required for analysis. This pragmatic advantage should also be realized in the generation of chromosomal rearrangements typically mediated by Cre-catalyzed recombination (Ramrez-Solis et al. 1995; Smith et al. 1995; Hérault et al. 1998) or in the condensation of tandem repeats resulting from the random integration of transgenes following pronuclear injection (Garrick et al. 1998). Linkage of tACE-Cre to a loxP site defining the desired deletion endpoint should greatly simplify these chromosomal engineering processes.

This technique should prove especially valuable in the generation of mice harboring conditionalmutant alleles (Rossant and McMahon 1999). The creation of such animals often takes advantage of either the Cre/loxP or FLP/FRT recombination systems to create genetic deletions regulated by the restricted spatial or temporal expression of the appropriate recombinase (Gu et al. 1994; Meyers et al. 1998). The recombinogenic elements, lox $P$ or FRT, must first be introduced into the genome by linkage to a selectable marker gene. Because it is essential that the ground state of such experiments be wild type, it is imperative that the marker gene not influence the expression of the target gene. If the two recombinase systems were employed in the same animal, for example, the self-excising cassette expressing FLP and deletion elements responding to the conditional expression of Cre, such a requirement could be met.

Self-excision strategies will undoubtedly find use outside the realm of genetically modified mice. New strains of agricultural crops are now equipped with 'terminator' genes to limit the propagation of proprietary traits (Service 1998). A self-excision mechanism activated only in the germ line would provide a single-step method to restrict those traits to a single, founding generation and may reduce the threat of unintended transmission of genetic traits to nontarget species. There are also proposals to attempt in utero human gene therapy as a means of correcting genetic deficiencies (Wadman 1998). Because such protocols will induce genetic changes in embryonic cells, including those that may colonize the germ line, they have raised both moral and pragmatic objections (Ewbank 1998; McLaren 1998). If, however, such modi-

Table 1. Genotypic analysis of progeny

\begin{tabular}{lcccc}
\hline \multirow{2}{*}{$\begin{array}{l}\text { Cell } \\
\text { line }\end{array}$} & $\begin{array}{c}\text { No. of } \\
\text { chimeras }\end{array}$ & \multicolumn{3}{c}{ Genotype of progeny } \\
\cline { 2 - 5 } & 3 & $+/+$ & $+/$ ACN & $+/$ lox \\
\hline 1d-7 & 9 & 37 & 0 & 26 \\
1h-9 & 1 & 7 & 0 & 32 \\
1f-9 & 13 & 67 & 0 & 71 \\
Total & &
\end{tabular}

Male chimeric animals derived from three cell lines were mated with C57Bl/6 females. DNA was extracted from tails of all agouti pups and was genotyped as described in Materials and Methods and the legend to Fig. 3. The number of animals in each genotype is indicated. 
fications were linked with a germ line-expressed recombinase and flanked with recombinogenic elements, the challenges to such modifications may be removed along with the intervening DNA (Capecchi 1999).

\section{Materials and methods}

Vector construction

The self-excision cassette was assembled into the bacterial plasmid pBS (Stratagene) using standard cloning methods. The $t A C E$ promoter sequences are nucleotides 495-1194; the Cre gene includes the entire protein-coding domain from pMCl-Cre (Gu et al. 1993) followed by the minimal poly(A) sequence from the HSV-TK gene (Thomas and Capecchi 1987); intron sequences from the SV40 t-antigen gene, nucleotides $4637-$ 4572, were amplified by PCR and inserted between codons 283 and 284 of $\mathrm{Cre}_{\text {; }}$ the $\mathrm{NeO}^{\mathrm{r}}$ gene is an 873-bp PstI-BamHI fragment isolated from pMClNeo-poly(A) (Thomas and Capecchi 1987); the promoter includes bases 1-713 from the mouse RNA polymerase II large subunit gene; the 34-bp minimal $\operatorname{lox} P$ sites are in parallel orientation at each end of the

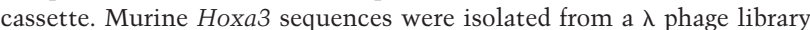
constructed in this laboratory of genomic DNA isolated from ES cells. Sequences used for the targeting vector extend from a Sau3AI site, $2.2 \mathrm{~kb}$ upstream of the ATG in exon 1 to an EcoRI site $5.5 \mathrm{~kb} \mathrm{3}$ ' of the TGA in exon 2. ACN was inserted into the BgIII site in the homeodomain in exon 2. An 8-bp EcoRV-containing oligonucleotide linker was also inserted at the Eco47III site in exon 1. This introduces a premature stop codon, creating an allele of Ноха3 to be used in future studies of this locus.

ES cells: transformation, screening, and blastocyst injection

The targeting vector $\mathrm{pRVa}{ }^{\mathrm{ACN}}$ was introduced in linear form by electroporation into RI ES cells (Nagy et al. 1993) that were subsequently selected for resistance to G418 and FIAU. Approximately $2 \times 10^{7}$ cells were subjected to electroporation and 144 drug-resistant colonies isolated. DNA was extracted from cells of each clone and subjected to analysis by Southern transfer under previously described conditions (Thomas and Capecchi 1987). Homologous recombination was verified following digestion with two separate restriction endonucleases and hybridization with three individual probes. No rearrangements other than the predicted homologous recombination reaction were seen nor were any homologous recombination events accompanied by detectable random integration of vector sequences. Cells from clones identified as heterozygous at the Ноха3 locus were injected into C57Bl/6-derived blastocysts that were allowed to come to term. Chimeric progeny were identified by coat color, and those males estimated to contain $>80 \%$ ES cell contribution were mated with $\mathrm{C} 57 \mathrm{Bl} / 6$ females.

Tissue and cell genotype analysis

DNA was extracted from tail biopsies of chimeric males and their progeny, as well as from tissues isolated from euthanized chimeric animals, and resuspended in TE buffer. Approximately $1 \mu \mathrm{g}$ of DNA was dissolved in $40 \mu \mathrm{l}$ of a PCR lysis buffer (Spyropoulos and Capecchi 1994), denatured at $95^{\circ} \mathrm{C}$ for $5 \mathrm{~min}$, and quick-chilled on ice. Five microliters of the denatured DNA solution was amplified for 30 cycles in a $25-\mu 1$ reaction mixture under previously described reaction conditions and cycling parameters (Deng et al. 1993). Primer sequences were as follows: Primer 1 , 5'-GCTCTTCCTCTCTGTGTCCTG-3', represents sequences $5^{\prime}$ of the splice acceptor site in the Hoxa3 intron (J. Greer and K. Thomas, unpubl.); primer 2, 5'-CGAATGCATAGAATTCAGATAGCC-3', is antisense sequence from Hoxa3, nucleotides 849-826 (McGinnis et al. 1984); primer 3, 5'-GCCTGCTTGCCGATTATCATGG-3', is from the sense strand of the $\mathrm{NeO}^{\mathrm{r}}$ gene, nucleotides 2121-2142. Amplified products were analyzed by electrophoresis through $3 \%$ NuSieve 3:1 agarose (FMC). Figure $3 \mathrm{~b}$ shows products from single, $25-\mu \mathrm{l}$ reactions; Figure $3 \mathrm{c}$ contains pools of 8 amplification reactions.

\section{GenBank accession numbers}

SV40 t-antigen (JO2400); loxP (M10287); RNA polymerase II large subunit (M14101); ACE (M61094); Neo (V00618).

\section{Acknowledgments}

We thank all members of the Capecchi laboratory's technical support group and the University of Utah Center of Excellence in Molecular Hematology for their expertise. Assistance from our colleagues in manuscript preparation and review was also greatly appreciated. J.G. was supported by the Dee Fellowship and a National Institutes of Health Genetics Training Grant; M.B. was supported by the University of Utah Center of Excellence in Molecular Hematology.

The publication costs of this article were defrayed in part by payment of page charges. This article must therefore be hereby marked 'advertisement' in accordance with 18 USC section 1734 solely to indicate this fact.

\section{References}

Araki, K., M. Araki, J.I. Miyazaki, and P. Vassalli. 1995. Site-specific recombination of a transgene in fertilized eggs by transient expression of Cre-recombinase. Proc. Nat1. Acad. Sci. 92: 160-164.

Broach, J.R. and J.B. Hicks. 1980. Replication and recombination functions associated with the yeast plasmid, $2 \mu$ circle. Cell 21: 501508.

Capecchi, M.R. 1999. Human germline gene therapy: A discussion on how and why. In Engineering the human germline (ed. G. Stock and J. Campbell), Oxford University Press, New York, NY. (In press.)

Colledge, W.H., B.S. Abella, K.W. Southern, R. Ratcliff, C. Jiang, S.H. Cheng, L.J. MacVinish, J.R. Anderson, A.W. Cuthbert, and M.J. Evans. 1995. Generation and characterization of a $\Delta$ F508 cystic fibrosis mouse model. Nat. Genet. 10: 445-452.

Deng, C., K.R. Thomas, and M.R. Capecchi. 1993. Location of crossovers during gene targeting with insertion and replacement vectors. Mol. Cell. Biol. 13: 2134-2140.

Ewbank, J. 1998. Problems of germline therapy. Nature 392: 645.

Garrick, D., S. Fiering, D.I.K. Martin, and E. Whitelaw. 1998. Repeatinduced gene silencing in mammals. Nat. Genet. 18: 56-59.

Gu, H., Y.-R. Zou, and K. Rajewsky. 1993. Independent control of immunoglobulin switch recombination at individual switch regions evidenced through Cre-loxP-mediated gene targeting. Cell 73: 11551164.

Gu, H., J.D. Marth, P.C. Orban, H. Mossmann, and K. Rajewsky. 1994. Deletion of a DNA polymerase beta gene segment in T cells using cell type-specific gene targeting. Science 265: 103-106.

Hasty, P., R. Ramírez-Solis, R. Krumlauf, and A. Bradley. 1991. Introduction of a subtle mutation into the Hox-2.6 locus in embryonic stem cells. Nature 350: 243-246.

Hérault, Y., M. Rassoulzadegan, F. Cuzin, and D. Duboule. 1998. Engineering chromosomes in mice through targeted meiotic recombination (TAMERE). Nat. Genet. 20: 381-384.

Langford, K.G., S.-Y. Shai, T.E. Howard, M.J. Kovac, P.A. Overbeek, and K.E. Bernstein. 1991. Transgenic mice demonstrate a testis-specific promoter for angiotensin-converting enzyme. J. Biol. Chem. 266: $15559-15562$.

McGinnis, W., C.P. Hart, W.J. Gehring, and F.H. Ruddle. 1984. Molecular cloning and chromosome mapping of a mouse DNA sequence homologous to homeotic genes of Drosophila. Cell 38: 675-680.

McLaren, A. 1998. Problems of germline therapy. Nature 392: 645.

Meyers, E.N., M. Lewandoski, and G.R. Martin. 1998. An Fgf8 mutant allelic series generated by Cre- and Flp-mediated recombination. Nat. Genet. 18: 136-141.

Nagy, A., J. Rossant, R. Nagy, W. Abramow-Newerly, and J.C. Roder. 1993. Derivation of completely cell culture-derived mice from early-passage embryonic stem cells. Proc. Natl. Acad. Sci. 90: 84248428.

O'Gorman, S., N.A. Dagenais, M. Qian, and Y. Marchuk. 1997. Protamine-Cre recombinase transgenes efficiently recombine target sequences in the male germ line of mice, but not in embryonic stem cells. Proc. Nat1. Acad. Sci. 94: 14602-14607.

Olson, E.N., H.-H. Arnold, P.W.J. Rigby, and B.J. Wold. 1996. Know your neighbors: Three phenotypes in null mutants of the myogenic bHLH gene MRF4. Cell 85: 1-4.

Pikaart, M.J., F. Recillas-Targa, and G. Felsenfeld. 1998. Loss of transcriptional activity of a transgene is accompanied by DNA methylation and histone deacetylation and is prevented by insulators. Genes \& Dev. 12: 2852-2862.

Ramírez-Solis, R., P. Liu, and A. Bradley. 1995. Chromosome engineering in mice. Nature 378: 720-724. 
Bunting et al.

Rossant, J. and A. McMahon. 1999. 'Cre'-ating mouse mutants-A meeting review on conditional mouse genetics. Genes \& Dev. 13: 142145 .

Schwenk, F., U. Baron, and K. Rajewsky. 1995. A cre-transgenic mouse strain for the ubiquitous deletion of loxP-flanked gene segments including deletion in germ cells. Nucleic Acids Res. 23: 5080-5081.

Service, R.F. 1998. Seed-sterilizing 'terminator technology' sows discord. Science 282: 850-851.

Smith, A.J.H., M.A.D. Sousa, B. Kwabi-Addo, A. Heppell-Parton, H. Impey, and P. Rabbitts. 1995. A site-directed chromosomal translocation induced in embryonic stem cells by Cre-lox $P$ recombination. Nat. Genet. 9: 376-385.

Spyropoulos, D.D. and M.R. Capecchi. 1994. Targeted disruption of the even-skipped gene, evx1, causes early postimplantation lethality of the mouse conceptus. Genes \& Dev. 8: 1949-1961.

Sternberg, N. and D. Hamilton. 1981. Bacteriophage P1 site-specific recombination. I. Recombination between loxP sites. J. Mol. Biol. 150: 467-486.

Thomas, K.R. and M.R. Capecchi. 1987. Site-directed mutagenesis by gene targeting in mouse embryo-derived stem cells. Cell 51: 503-512.

Wadman, M. 1998. US gene-therapy proposals come under fire. Nature 395: 309 


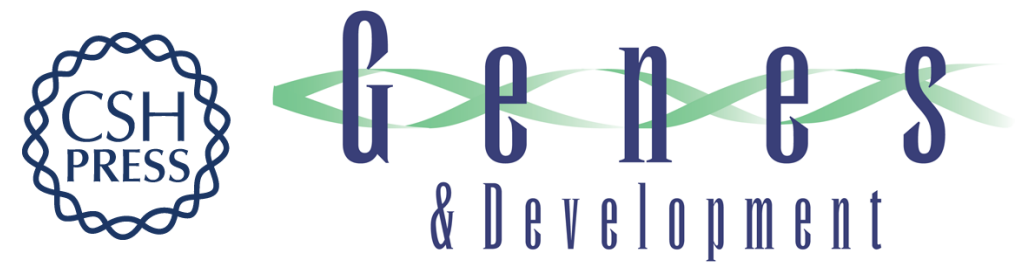

\section{Targeting genes for self-excision in the germ line}

Michaeline Bunting, Kenneth E. Bernstein, Joy M. Greer, et al.

Genes Dev. 1999, 13:

References This article cites 26 articles, 10 of which can be accessed free at: http://genesdev.cshlp.org/content/13/12/1524.full.html\#ref-list-1

License

Email Alerting

Receive free email alerts when new articles cite this article - sign up in the box at the top Service right corner of the article or click here.

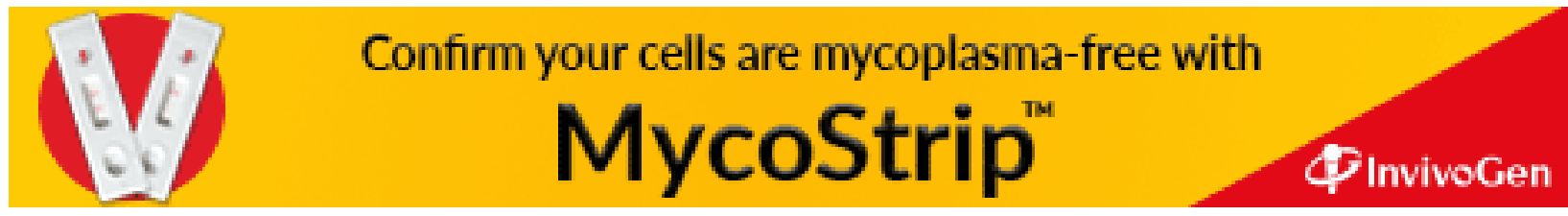

